\title{
Exclusion criteria and outcome in pressure reduction of intussusception
}

\author{
D den Hollander, D M Burge
}

\begin{abstract}
Experience of 53 episodes of intussusception was examined to compare the observed success rate of pressure reduction with potential outcome had stricter exclusion criteria been applied (history $>24$ hours, presence of rectal bleeding, radiological signs of intestinal obstruction). With stricter criteria 25 avoidable laparotomies would have been performed. Most infants can be cured of intussusception by pressure reduction and though some must be excluded this decision should be based on clinical assessment by those experienced in its management. Pressure reduction should not be attempted in the absence of a surgeon with regular experience of intussusception.
\end{abstract}

(Arch Dis Child 1993;68:79-81)

The treatment options for a child with intussusception are those of pressure reduction, laparotomy with manual reduction or resection. Pressure reduction, be it with barium or air, is a highly effective method of treatment with success rates of up to $80 \% .^{1}$ Although there are circumstances where it would be dangerous to attempt pressure reduction, controversy exists about specific contraindications. Although some paediatric surgical centres have reported increased success with pressure reduction after relaxing their exclusion criteria, ${ }^{2}$ two reports in the surgical literature suggest that exclusion criteria for pressure reduction should be more strict. ${ }^{3}{ }^{4}$ In particular it has been suggested that children with a history longer than 24 hours, who have had rectal bleeding, or who have signs of intestinal obstruction should not have pressure reduction attempted. In many, if not most paediatric units, intussusception will be managed by paediatricians and general surgeons. The latter might be influenced in their decisions regarding indications for surgery by such publications. It was our impression that adoption of such strict criteria will result in the performance of a number of avoidable laparotomies. We therefore undertook this study of our own series of patients with intussusception in whom pressure reduction was attempted, to see how adoption of these criteria might have affected their management.

\section{Patients and methods}

In this retrospective study we reviewed the records of all children aged 2 years or less presenting to the Wessex Regional Centre for
Paediatric Surgery with proved intussusception over a four year period. Particular note was made of the length of history at presentation, the presence or absence of rectal bleeding, the clinical findings recorded on examination, and radiological features. The length of history was calculated from the onset of symptoms suggestive of intussusception rather than the onset of symptoms of any preceeding viral illness. Attempted pressure reduction was performed using barium or, more recently, air. ${ }^{5}$

Radiological evidence of intestinal perforation is an absolute indication for surgery. Exclusion from attempted pressure reduction in our centre in the absence of perforation is based on a clinical assessment of the child taking into account evidence of peritonitis, peritonism, or the presence of clinical shock that failed to respond quickly to colloid replacement.

\section{Results}

Fifty two children were admitted with 55 proved episodes of intussusception. Two children were declared unsuitable for pressure reduction on clinical grounds as described above and both underwent primary laparotomy. Pressure reduction was attempted in 53 episodes in 50 children and was successful at the first attempt in $31(58 \%)$. Eleven episodes where initial pressure reduction failed underwent a second attempted pressure reduction that was successful in four, bringing the overall success rate to $66 \%$. Attempted pressure reduction was deemed to have been unsuccessful in 18 episodes (34\%), all of which required laparotomy. Six of these children underwent intestinal resection.

Attempted pressure reduction was undertaken using barium in 36 cases, barium and air in three cases, and air alone in 14 cases. Success rates for barium and air alone were $76 \%$ and $64 \%$ respectively.

Three children who underwent attempted pressure reduction sustained intestinal perforation and all underwent surgery. Two of them had leakage of barium noted during screening. In the first of these barium ran freely out of a colonic perforation without the use of pressure. There had been no radiological evidence of perforation before this and at laparotomy a well established perforation was found to be plugged with the tip of the intussuscepting bowel. This perforation was not felt to be directly due to the use of pressure as none was used. The second perforation occurred after 90 seconds of pressure in a child with a four day history of intussusception. The third perforation was detected as a small quantity of barium noted adjacent to the 
Outcome of pressure reduction in relation to length of history in 53 episodes of intussusception

\begin{tabular}{lrc}
\hline $\begin{array}{l}\text { Duration of symptoms } \\
\text { (hours) }\end{array}$ & Success $(\%)$ & Failure \\
\hline$<24$ & $15(88)$ & 2 \\
$25-48$ & $8(89)$ & 1 \\
All patients $<48$ & $23(88)$ & 3 \\
$49-72$ & $7(64)$ & 4 \\
All patients $<72$ & $30(81)$ & 7 \\
$>72$ & $4(25)$ & 12 \\
\hline
\end{tabular}

intussusception at laparotomy after failed pressure reduction and had not been suspected preoperatively. In each of these cases the decision to proceed with pressure reduction had been made by a paediatric surgical registrar and in each case the consultant surgeon subsequently commented that the child should not have been offered pressure reduction based on our existing exclusion criteria. There were no deaths in the series and all children made a full recovery.

A high success rate for attempted pressure reduction was achieved regardless of whether patients presented within the first 24 or 48 hours (table). After 72 hours, however, the chances of successful pressure reduction were greatly reduced. Had we excluded all children with a history of greater than 24 hours we would have performed an additional 19 laparotomies.

Blood was either passed by rectum or was visible on the glove after rectal examination in $33 / 53$ cases. Successful pressure reduction occurred in 20 of these $(61 \%)$ compared with $17 / 20$ cases $(85 \%)$ where no rectal bleeding occurred (not significant). All three children with perforation had had rectal bleeding.

Signs of intestinal obstruction were apparent on plain radiography in 10 children, half of whom had successful pressure reduction. Two of the children with perforation had radiological obstruction but one did not.

Had we adopted the above exclusion criteria only 11 of the 53 episodes in these series would have been offered pressure reduction and this would have been successful in 10. An unnecessary laparotomy would have been performed in 25 patients.

\section{Discussion}

Pressure reduction of intussusception, as introduced by Hirschsprung in 1876 and popularised by Ravich in 1948, has clear advantages over operative treatment. Exclusion criteria and results vary greatly between different centres. In a series of 125 children from the Royal Hospital for Sick Children in Edinburgh, published in $1988,{ }^{3}$ pressure reduction was offered to $61 \%$ of children and was successful in $38 \%$ (or $23 \%$ of the whole group). Children were excluded from attempted pressure reduction if they had had symptoms for longer than 24 hours, were clinically shocked or dehydrated, showed evidence of peritonitis or gross intestinal obstruction, or had had rectal bleeding.

In a report from Manchester pressure reduction was offered in $69 \%$ of 78 episodes of intussusception and was successful in 50\% ( $35 \%$ of the whole group). ${ }^{6}$ Length of history greater than 48 hours, the presence of rectal bleeding, peritonism, or recurrence of intussusception after previous pressure reduction were used as exclusion criteria in some patients. In a report from Carshalton, pressure reduction was performed in 34 of 75 patients and was successful in only six (8\% of the whole group). ${ }^{4}$ Although a complete list of exclusion criteria for this centre was not given, length of history greater than 48 hours and signs of intestinal obstruction on abdominal $x$ ray film are mentioned as indications to perform a primary laparotomy.

We attempted pressure reduction in 53 of 55 episodes (96\%). Had we adopted such strict exclusion criteria for pressure reduction in our series only 11 of our patients would have been offered pressure reduction and 10 of these would have been successful. Twenty five children would have been subjected to unnecessary laparotomy raising our laparotomy rate from $34 \%$ to $80 \%$. The price that we have paid for our attitude to pressure reduction has been the development of three perforations. All of these children had presented with symptoms longer than 72 hours and when reviewed retrospectively during their admissions it was felt that all three children should have been excluded from attempted pressure reduction by our existing exclusion criteria.

Great care must still be excercised in the selection of children with intussusception for attempted pressure reduction and there is no substitute for clinical experience in making this decision. Perforation is an absolute contraindication for pressure reduction. The presence of bowel infarction within the intussusception should also preclude pressure reduction and it is in the assessment of this feature that clinical experience is required. In addition relative exclusion criteria may pertain in individual centres depending on the availability of a radiologist experienced in pressure reduction. Certainly pressure reduction should not be attempted in the absence of a suitably experienced surgical and anaesthetic team standing by on site should it be unsuccessful or perforation occur. We recommend that a second attempt at pressure reduction should not be undertaken outside a specialist paediatric surgical unit.

We accept that the majority of children with intussusception will be admitted under the care of paediatricians who do not have access within their hospital either to specialist paediatric surgeons or to radiologists with extensive experience of pressure reduction. The quality of radiological and surgical intervention will depend on the experience of the specialists involved; indeed it is the recommendation of the report of the National Confidential Enquiry into Perioperative Deaths that surgery in infants should not be undertaken on an occasional basis. ${ }^{7}$ It is clearly the responsibility of paediatricians to be aware of the results of attempted pressure reduction in their unit and the laparotomy criteria adopted by local surgeons in comparison with the results in specialist centres. Our results indicate that the adoption of strict exclusion 
criteria will deny a significant proportion of children the opportunity of cure without resort to laparotomy.

We would like to thank Mr J D Atwell, Mr N V Freeman, and Mr D M Griffiths for permission to report their cases.

1 Mackay A J, MacKeller A, Sprague P. Intussusception in children, a review of 91 cases. Aust $N Z \mathcal{J}$ Surg 1987;57: $15-7$
2 Ein S H, Mercer S, Humphry A, McDonald P. Colonic perforation during attempted barium enema reduction of intussusception. F Pediatr Surg 1981;16:313-5.

3 Wilson-Storey D, MacKinlay G A. Intussusception: a surgical condition. $\mathcal{R}$ Coll Surg Edinb 1988;33:270-3.

4 Mann D W K, Heath A L, Eckstein H B. Intussusception in infancy and childhood. A 13 year review of 75 patients. $Z$ Kinderchir 1983;38:383-6.

5 Paes R A, Hyde I, Griffiths D M. The management of intussusception. Br f Radiol 1988;61:187-9.

6 Winstanley J H R, Doig C M, Brydon H. Intussusception: the case for barium reduction $\mathcal{F} R$ Coll Surg Edinb 1987;32: the case 7 .

7 Campling E A, Devlin H B, Lunn J N. Report of the national confidential enquiry into perioperative deaths. London: HMSO, 1989

Girl meets boy; boy meets girl; and measles

'Are males weaker or do their sisters talk too much?' The title of a 1983 paper $^{1}$ seems to suggest a certain facility for de Bono-style lateral thinking. ${ }^{2}$ Peter Aaby, an epidemiologist from Copenhagen, has been studying patterns of measles transmission in various parts of Africa including the Gambia, Guinea-Bissau, Senegal, and Kenya and has noticed that measles is more severe if contracted from somebody of the opposite sex. A paper in the Lancet (Peter Aaby, 1992;340:388-91) gives data from a study done in a rural area of Senegal between 1983 and 1986. The case fatality rate (CFR) for measles was highest at the age of 2 years at which age the CFR for boys was $12 \%$ and for girls $15 \%$. Children infected within a household were twice as likely to die as children infected from outside the household (CFR $8 \% v 4 \%$ ) presumably because of heavier exposure. Cross sex transmission was more dangerous than same sex transmission. Boy to boy transmission was associated with a CFR of $4 \cdot 0 \%$. CFRs (relative risk $[95 \%$ confidence interval] $)$ for other modes of transmission were: girl to girl $5.8(1.4[0.6$ to 3.4$])$, boy to girl $11.4(2.8[1.4$ to 5.7$])$, and girl to boy $12.9(2.9[1.4$ to 6.0$])$. The effect of cross sex transmission was greater if infecter and infectee both lived in the same household (ate together), or hut (sleeping quarters) rather than just in the same compound. A study of twins in Guinea-Bissau showed a higher mortality when they were of different sex (relative risk $1 \cdot 47[1 \cdot 14$ to $1 \cdot 90]),{ }^{3}$ and in a case-control study the older sibling nearest in age was of the opposite sex in $74 \%$ of children who died of measles and in $46 \%$ of controls. In a different part of Senegal families with two children were studied. ${ }^{4}$ Measles mortality was greater in children with a sibling of different sex (relative risk $1 \cdot 81[1 \cdot 17$ to $2 \cdot 82]$ ).

What is the explanation for this strange observation? It does not appear to be a peculiarity of life in Senegal as a similar phenomenon has been observed in other parts of West Africa, in East Africa, and in early twentieth century Copenhagen. It may not be specific to measles as the author claims to have data showing that it may apply to chickenpox but not to whooping cough. The most likely theory is that cross sex transmission involves a bigger dose of virus, although there is no evidence from observed behaviour patterns in children of this age of closer cross sex contact. The alternative possibility, that the virulence of the virus is in some way increased by passage through the cells of somebody of the opposite sex, would presumably pose a much greater challenge to microbiological theorists and is therefore more interesting but less probable.

ARCHIVIST

1 Aaby P, Bukh J, Lisse IM, Smits AJ. Les hommes sont-ils plus faibles ou leurs soeurs parlent-elles trop? Essai sur la transmission des maladies infectieuses. Anthrop Soc $1983 ; 7: 47-59$.

2 de Bono E. Lateral thinking. London: Penguin, 1990.

Aaby $\mathrm{P}$, Mølbak K. Siblings of opposite sex as a risk factor for child mortality. BMF 1990;301:143-5.

4 Pison G, Aaby P, Knudsen $K$. Increased risk of death from measles in children with a sibling of opposite sex in Senegal. BMF 1992;304:284-7. 